\title{
Étude immuno-chimique de l'hémolymphe d'abeille ouvrière adulte (Apis mellifera $L$ ) saine ou infestée par Varroa jacobsoni Oud
}

\author{
JP Dandeu 1, M Lux 1, ME Colin 2, J Rabillon 1, B David 1 \\ 1 Institut Pasteur, Laboratoire d'immuno-chimie des acariens et des venins d'hyménoptères, \\ unité d'immuno-allergie, 28 rue du Dr Roux, 75724 Paris Cedex 15; \\ 2 INRA, station de zoologie et d'apidologie, domaine Saint-Paul-Cantarel, \\ route de Marseille, BP 91, 84140 Montfavet, France
}

(Reçu le 12 janvier 1990; accepté le 5 décembre 1990)

\begin{abstract}
Résumé - Varroa jacobsoni Oud, ectoparasite de l'abeille Apis mellifica $L$, se nourrit essentiellement par succion de l'hémolymphe de l'hôte, larve ou insecte adulte, à travers la cuticule. La composition antigénique de cette hémolymphe peut varier, soit du fait de la ponction elle-même, soit de l'action des enzymes injectées, et les modifications peuvent donc être quantitatives ou qualitatives. Nous montrons ici que des marqueurs antigéniques qui semblent ne pas provenir de l'acarien luimême, peuvent apparaître spécifiquement après l'infestation même récente de la ruche par celui-ci, sans préjuger qu'ils résultent ou non d'une réaction spécifique à la varroatose ou au simple traumatisme occasionné par la morsure de l'acarien responsable.
\end{abstract}

Varroa jacobsoni / Apis mellifera / hémolymphe / immunochimie / immuno-électrophorèse bidimensionnelle

\section{INTRODUCTION}

Varroa jacobsoni Oud (Acari, Varroidae) est un ectoparasite de l'abeille Apis mellifera L. La varroatose est probablement la plus importante des maladies des ruchers, qui risque de détruire à relativement court terme l'ensemble du cheptel apicole mondial. Cet acarien fut décrit pour la première fois par Oudemans (1904).
Le Varroa femelle se nourrit par ponction de l'hémolymphe de l'insecte et plus particulièrement durant les premiers stades de l'ontogénèse provoquant ainsi des malformations chez les insectes adultes qui ont survécu (Ifantidis, 1983; Colin, 1982; Lux, 1987, 1988).

Si la composition de l'hémolymphe de l'abeille saine a été étudiée par Gilliam et Jeter (1970) et Gilliam et Mac Caughey (1972) ainsi que les modifications entraî- 
nées par une infestation bactérienne, telles que l'apparition de molécules agglutinantes, Glinsky et Jarosz en 1984 ont montré par électrophorèse en gel d'acrylamide, une altération des protéines de l'hémolymphe de larve d'abeille mâle parasitée par Varroa jacobsoni, mais aucune étude immunologique de l'hémolymphe de l'abeille ouvrière adulte parasitée par Varroa jacobsoni n'a été réalisée jusqu'ici.

Pour l'étude immunochimique préliminaire dont nous présentons ici les résultats, nous avons choisi de récolter et de préparer des pools d'hémolymphe d'abeilles adultes ayant 7-8 jours, provenant de ruches infestées, d'un même rucher durant des temps variables, par Varroa jacobsoni, la comparaison étant faite avec un pool d'hémolymphe d'abeilles du même âge, provenant du plusieurs ruches du même rucher exempt de tout acarien Varroa jacobsoni.

\section{MATÉRIEL ET MÉTHODES}

\section{Recherche des acariens \\ Varroa jacobsoni dans les ruchers}

La recherche des acariens Varroa jacobsoni dans les ruches a été effectuée en utilisant un aérosol répandu à l'aide de l'appareil EDAR avec le - $\mathrm{N}$-méthyl bis- (2,4 - xylyliminométhyl) amine (Amitraze) puis recueil et comptage des acariens sur un lange gras.

\section{Récolte des hémolymphes}

L'hémolymphe a été recueillie par ponction dans le cœur dorsal de l'abeille en introduisant un tube capillaire en verre entre le troisième et le quatrième segment abdominal visible. Environ $10 \mu \mathrm{l}$ par insecte ont été recueillis et immédiatement lyophilisés. Les échantillons étudiés sont des pools des prélèvements effectués sur un centaine d'abeilles ouvrières adultes et sont désignés comme suit :
- HS : hémolymphe d'abeilles provenant d'un rucher sain (Grimaux, Var, France) 1985;

- $\mathrm{HP}_{1}$ : hémolymphe d'abeilles provenant d'un rucher infesté par Varroa jacobsoni depuis un an (Draguignan, Var, France) 1985;

- $\mathrm{HP}_{2}$ : hémolymphe d'abeilles provenant d'une ruche (Maine et Loire) considérée comme saine lors du prélèvement en août 1986 et dont l'infestation n'a été diagnostiquée que 3 mois plus tard.

Le contrôle a été effectué selon le procédé décrit ci-dessus.

\section{Extrait d'acariens Varroa jacobsoni}

Afin de déterminer la présence éventuelle dans ces hémolymphes, d'antigènes provenant de l'acarien, un extrait a été préparé à partir de Varroa jacobsoni récoltés sur la cuticule des abeilles infestées. Ces acariens ont été lavés afin d'éliminer toute contamination par l'hémolymphe ingérée ou adsorbée (Tewarson, 1981; Tewarson et Engels, 1982).

L'extraction a été faite par un broyage en milieu aqueux à $\mathrm{pH}$ neutre suivie d'une centrifugation à $10000 \mathrm{Rpm} / \mathrm{min}$, le surnageant dénommé EBVarj a été conservé par congélation à $-20^{\circ} \mathrm{C}$.

\section{Techniques immunochimiques}

L'étude comparée de l'hémolymphe d'abeille saine ou parasitée par Varroa jacobsoni a été réalisée grâce à l'utilisation des méthodes immunochimiques décrites par Axelsen et al (1973) et Axelsen (1975),c'est-à-dire l'immuno electrophorèse bidimensionnelle avec gel intermédiaire vierge ou contenant des anticorps. Les anticorps utilisés ont été obtenus par relargage au $\mathrm{Na}_{2} \mathrm{SO}_{4}$ à partir des pools de sérums de lapins hyperimmunisés, soit contre HS, soit contre $H P_{1}$.

Les anticorps anti-HS proviennent d'un pool de sérum de lapins hyperimmunisés. La première électrophorèse est pratiquée avec $10 \mu \mathrm{g}$

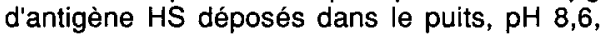
avec $20 \mathrm{~V} / \mathrm{cm}$, à $10^{\circ} \mathrm{C}$ pendant $25 \mathrm{~min}$.

Après transfert du gel de première dimension sur le support plastique, on coule le gel intermé- 
diaire vierge ou contenant des anticorps. Pour la deuxième dimension, les anticorps de lapin antiHS sont mélangés au gel d'agarose qui est coulé sur la partie anodique restante du support $\left(2,5 \mu \mathrm{l} / \mathrm{cm}^{2}\right.$ d'anticorps sont utilisés dans le cas de I'HS et $25 \mu \mathrm{l} / \mathrm{cm}^{2}$ dans le cas de l'HP). Cette différence est due au fait que les anticorps antiHS sont plus concentrés que les anticorps antiHP. La deuxième électrophorèse est pratiquée perpendiculairement à la première, à $7,5 \mathrm{~V} / \mathrm{cm}$, à $10^{\circ} \mathrm{C}$ pendant $18 \mathrm{~h}$.

\section{RÉSULTATS ET DISCUSSION}

Par immuno-électrophorèse bidimensionnelle, nous avons pu mettre en évidence 9 constituants antigéniques différents dans I'hémolymphe d'abeilles ouvrières saines (HS) (fig 1a, b) et 13 dans les hémolymphes d'abeilles ouvrières parasitées $\left(H P_{1}\right)$ (fig 2a, b). Comme contrôle l'extrait d'acarien EB Var $j$ a été mis à réagir avec les anticorps anti-HS d'une part et les anticorps anti-HP 1 d'autre part. Aucune réaction de précipitation n'a pu être révélée dans les conditions de l'expérience.

Afin de prouver que les 4 constituants antigéniques supplémentaires révélés dans $\mathrm{HP}_{1}$ sont des marqueurs antigéniques spécifiques de la parasitose, une im-
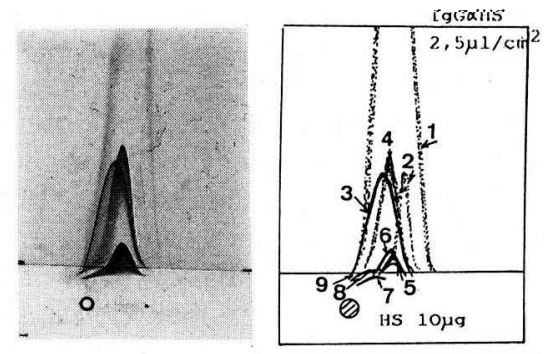

Fig 1. a Immuno-électrophorèse bidimensionnelle de l'hémolymphe d'abeille ouvrière adulte saine HS. b Schéma de la figure 1a, avec la numérotation des différents antigènes révélés dans HS par le sérum de lapin anti-HS. muno-électrophorèse bidimensionnelle avec un gel intermédiaire a été réalisée. En première dimension, les hémolymphes $H P_{1}$ et $\mathrm{HP}_{2}$ ont été séparées par électrophorèse de zone et mises à réagir avec les anticorps homologues (anti-HP $\mathrm{P}_{1}$ ).

En deuxième dimension, le gel intermédiaire contenait les anticorps antihémolymphe d'abeille saine (anti-HS) à une concentration préalablement définie pour retenir tous les antigènes présents dans HS. Ainsi sur les figures $3 a$, b et $4 a$, $b$, on peut voir qu'au moins 3 des 4 antigènes supplémentaires dont l'existence a été constatée dans $\mathrm{HP}_{1}$ et $\mathrm{HP} \mathrm{P}_{2}$ peuvent être considérés comme des marqueurs spécifiques de la varroatose, ou plus exactement de ses effets sur l'hémolymphe des abeilles ouvrières adultes. Ces mêmes marqueurs ont pu être détectés dans l'hémolymphe des abeilles présumées saines $\mathrm{HP}_{2}$ provenant d'une ruche où la présence de Varroa jacobsoni n'a pu être révélée par les tests classiques que 3 mois plus tard.

Compte tenu des résultats obtenus avec les témoins encagés (abeilles adultes saines), il est à noter que ce simple stress provoque des modifications du profil protéique. II n'a pas été possible de mettre en
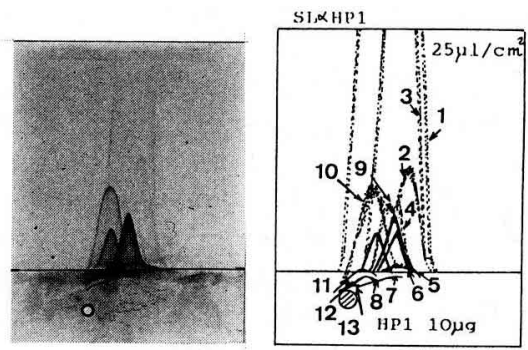

Fig 2. a Immuno-électrophorèse bidimensionnelle de l'hémolymphe d'abeille ouvrière adulte parasitée par Varroa jacobsoni HP . b Schéma de la figure 2a, avec la numérotation des différents antigènes révélés dans $\mathrm{HP}_{1}$ par le sérum de lapin anti-HP 1 . 

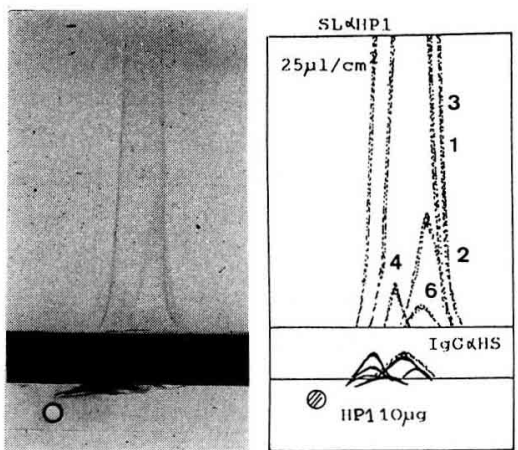

Fig 3. a Immuno-électrophorèse bidimensionnelle de l'hémolymphe d'abeille parasitée $\mathrm{HP}_{1}$ $(10 \mu \mathrm{g})$, avec gel intermédiaire contenant $0,6 \mathrm{ml}$ d'IgG-anti-HS. b Schéma de la figure $3 a$, avec la numérotation des antigènes spécifiques à $\mathrm{HP}_{1}$.

évidence des modifications supplémentaires dues à un traumatisme provoqué par une piqûre d'aiguille.

La possibilité que les marqueurs de la varroatose, dont la présence est suggérée par les résultats rapportés ici, aient une quelconque relation avec les peptides antibactériens mis en évidence par d'autres auteurs (Gilliam et Jeter 1970 et Casteels et al, 1989) reste à démontrer. Ceci pourrait être envisagé en recherchant une communauté antigénique entre ces derniers et les marqueurs décrits ici. Néanmoins la confirmation de nos résultats grâce à une étude systématique d'un grand nombre d'échantillons d'hémolymphe d'abeilles saines ou parasitées reste la condition sine qua non pour permettre la mise au point d'un diagnostic précoce de la varroatose.

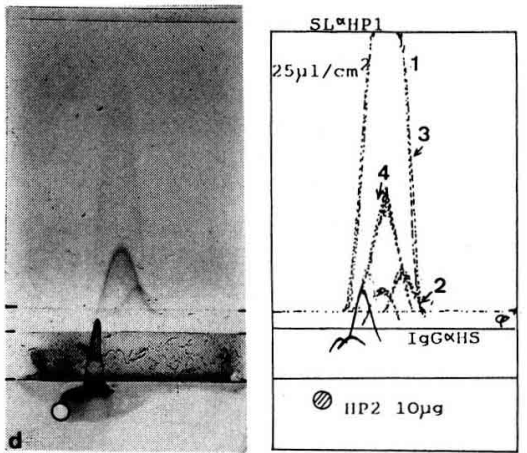

Fig 4. a Immuno-électrophorèse bidimensionnelle de l'hémolymphe d'abeille présumée saine $\mathrm{HP}_{2}(10 \mu \mathrm{g})$, avec gel intermédiaire contenant $0,6 \mathrm{ml} \mathrm{d}^{\prime} \mathrm{lgG}$-anti-HS. b Schéma de la figure $4 \mathrm{a}$, avec la numérotation des différents antigènes spécifiques à $\mathrm{HP}_{1}$.

\section{REMERCIEMENTS}

Cette étude a été réalisée avec le concours d'Intermiel (Programme de recherche sur la varroatose).

Summary - Immunochemical study of hemolymphs from healthy and Varroa jacobsoni Oud, infested adult workerbees (Apis mellifera L). Varroa jacobsoni Oud, an ectoparasite of the honey bee Apis mellifera $\mathrm{L}$ essentially feeds by sucking hemolymph through the cuticle of adult insect or larvae. Antigenic composition of hemolymph may be quantitatively or qualitatively modified either through an enzymatic process or as a direct result of the puncture. We show here that specific antigenic markers, which do not come from the mite itself, appear even after early in- 
festation of the hive. This was determined using immunochemical methods such as crossed immunoelectrophoresis and crossed line immunoelectrophoresis where rabbit IgG antibodies raised against hemolymph, either healthy or infested, were involved in homologous or heterologous reactions. Several antigens were defined and are currently under study. We have not yet been able to determine whether these markers result from a specific reaction to Varroa jacobsoni infestation or only from the traumatism due to the bite of this mite.

Varroa jacobsoni / Apis mellifera / hemolymph immunochemistry / crossedline immunoelectrophoresis

\footnotetext{
Zusammenfassung - Immunchemische Untersuchung der Hämolymphe von erwachsenen gesunden und von Varroa jacobsoni befallenen Arbeitsbienen (Apis mellifera). Varroa jacobsoni Oud, ein Ektoparasit der Honigbiene Apis mellifera $\mathrm{L}$, ernährt sich vor allem durch Saugen von Hämolymphe durch die Cuticula von erwachsenen Insekten oder Larven. Der Antigen-Komplex der Hämolymphe könnte quantitativ oder qualitativ verändert werden, entweder durch einen enzymatischen Proze $B$ oder als direkte Folge der Punktion. Wir zeigen hier, daß selbst in einem frühen Befallsstadium des Volkes charakteristische Antigen-Marker spezifisch aufzutreten scheinen. Diese Marker kommen nicht von der Milbe selbst. Das wurde nachgewiesen durch Anwendung von immunchemischen Methoden wie Kreuz-Immunelektrophorese und Kreuzlinien-Immunelektrophorese, wo Kaninchen IgG-Antikörper gegen Hämolymphe von gesunden oder befallenen Tieren erzeugten, zu homologen oder heterologen Reaktionen verwendet wurden. Es wurden mehrere Antigene be-
}

stimmt, die gegenwärtig in näherer Untersuchung stehen.

Entstehen diese Marker als Folge einer spezifischen Reaktion auf den Befall durch Varroa jacobsoni oder bloß als Folge der Verletzung durch den Biß dieser Milbe ? Wir waren noch nicht in der Lage, diese Frage zu beantworten.

Varroa jacobsoni / Apis mellifera / Hämolymphe / Immunchemie / KreuzlinienImmunelektrophorese

\section{RÉFÉRENCES}

Axelsen NH (1975) Quantitative immunoelectrophoresis: new developments and applications. Scand $J$ Immunol suppl 2

Axelsen NH, Kroll J, Weeke B (1973) A manual of quantitative immunoelectrophoresis. Methods and applications. Scand $J$ Immunol suppl 1

Casteels P, Ampe $C$, Jacobs $F$, Vaeck $M$, Tempst $P$ (1989) Apidaecins : antibacterial peptides trom honeybees. EMBO J 8, 23872391

Colin ME (1982) La Varroatose. Point Vét 14, (69), 21-28

Gilliam M, Jeter WS (1970) Synthesis of agglutinating substances in adult honeybees against Bacillus larvae. J Invertebr Pathol 16, 69-70

Gilliam M, Mac Caughey WF (1972) Total amino acids in developing worker honey bees (Apis mellifera L). Experientia 15, 142-143

Glinski Z, Jarosz J (1984) Alterations in haemolymph proteins of drone honey bee larvae parasitized by Varroa jacobsoni. Apidologie 15, 329-338

Ifantidis MD (1983) Ontogenesis of the Mite Varroa jacobsoni in worker and drone honeybee brood cells. J Apic Res 22, 200-206

Lux M (1987) Varroa jacobsoni Oudemans ectoparasite de l'abeille Apis mellifica Linné. Etude physico-chimique et immuno-chimique de l'hémolymphe d'abeille saine ou parasitée. Thèse de l'école pratique des hautes études, Montpellier 
Lux M (1988) Un regard neuf sur Varroa jacobsoni. Rev Fr Apic 480, 543-549

Oudemans AC (1904) A new genus and species of parasitic acari. Notes Zool Museum Leyden note VIII, 216-222

Tewarson NC (1981) Immunologische Untersuchungen über die Rolle von Hämolymph Pro- teinen der Honigbiene für Ernährung und Fortpflanzung von Varroa jacobsoni. Proc Symp Diagnose und Therapie der Varroatose Oberursel (Germany) 29 sept - 1 oct 1980; Bucharest, Apimondia, 39-47

Tewarson NC, Engels (1982) Undigested uptake of non-host proteins by Varroa jacobsoni. J Apic Res 21, 222-225 